\title{
A Functional Connectivity Inspired Approach to Non-Local fMRI Analysis
}

Anders Eklund, Mats Andersson and Hans Knutsson

\section{Linköping University Post Print}

N.B.: When citing this work, cite the original article.

(C)2012 IEEE. Personal use of this material is permitted. However, permission to reprint/republish this material for advertising or promotional purposes or for creating new collective works for resale or redistribution to servers or lists, or to reuse any copyrighted component of this work in other works must be obtained from the IEEE.

Anders Eklund, Mats Andersson and Hans Knutsson, A Functional Connectivity Inspired Approach to Non-Local fMRI Analysis, 2012, Proceedings of the 19th IEEE International Conference on Image Processing (ICIP), 2012, 1245-1248.

http://dx.doi.org/10.1109/ICIP.2012.6467092

Postprint available at: Linköping University Electronic Press

http://urn.kb.se/resolve?urn=urn:nbn:se:liu:diva-76119 


\title{
A FUNCTIONAL CONNECTIVITY INSPIRED APPROACH TO NON-LOCAL FMRI ANALYSIS
}

\author{
Anders Eklund ${ }^{a, b, c}$, Mats Andersson ${ }^{a, b}$, Hans Knutsson ${ }^{a, b}$ \\ ${ }^{a}$ Division of Medical Informatics, Department of Biomedical Engineering, Linköping University, Sweden \\ ${ }^{b}$ Center for Medical Image Science and Visualization (CMIV), Linköping University, Sweden \\ ${ }^{c}$ Department of Medical Imaging, University of Toronto, Canada
}

\begin{abstract}
We propose non-local analysis of functional magnetic resonance imaging (fMRI) data in order to detect more brain activity. Our non-local approach combines the ideas of regular fMRI analysis with those of functional connectivity analysis, and was inspired by the non-local means algorithm that commonly is used for image denoising. We extend canonical correlation analysis (CCA) based fMRI analysis to handle more than one activity area, such that information from different parts of the brain can be combined. Our non-local approach is compared to fMRI analysis by the general linear model (GLM) and local CCA, by using simulated as well as real data.
\end{abstract}

Index Terms - fMRI, non-local, CCA, functional connectivity, GPU

\section{INTRODUCTION}

It is a well known fact that many parts of the brain work together to solve a given task. Motor tasks often result in bilateral activation of the primary motor cortex, the supplementary motor area and the somatosensory cortex. A reading task normally results in activation of the visual cortex, Wernicke's area and Broca's area. Despite this fact, present methods for analysis of functional magnetic resonance imaging (fMRI) data are normally local. We therefore propose to combine information from different parts of the brain, in contrast to only using local averaging, to detect more brain activity.

It has been mentioned [1] that linear multi-voxel pattern analysis (MVPA) approaches to fMRI analysis [2, 3, 4] only use information from neighbouring voxels, and are thereby blind to non-local connections. One way to solve this problem is to use all the brain voxels in the analysis and, for example, calculate a weight that represents how important each voxel is for a classifier. If we however know that there only is activity in two areas, to use information from the whole brain will give a sub-optimal result. Consider a similar comparison, if we know the size of an activity area we also know the optimal filter, according to the matched filter theorem, to use a different filter would give worse results.
In this work we present an approach for non-local fMRI analysis, that combines the ideas for functional connectivity analysis [5] and regular fMRI analysis. Our work is based on the previous work on using canonical correlation analysis (CCA) [6] for fMRI analysis [7, 8, 9]. The algorithm is also inspired by the non-local means (NLM) algorithm [10] that is commonly used for image denoising. The main idea of NLM is to average pixels that are local in a feature space, rather than to average pixels that are local in a spatial sense. We will refer to our new approach for non-local CCA as NL CCA.

\section{METHODS}

\subsection{Non-local CCA}

The most common approach for statistical analysis of fMRI data is to apply the general linear model independently (GLM) to each voxel timeseries separately and then calculate a t-test or a F-test value [11]. One statistical approach that provides more adaptivity to the data is CCA [6]. While the GLM works with one multidimensional variable (e.g. temporal basis functions [11]), CCA works with two multidimensional variables (e.g. temporal and spatial basis functions $[7,8])$. The canonical correlation is defined as

$$
\rho=\operatorname{Corr}\left(\boldsymbol{\beta}^{\mathrm{T}} \boldsymbol{x}, \boldsymbol{\gamma}^{\mathrm{T}} \boldsymbol{y}\right)=\frac{\boldsymbol{\beta}^{\mathrm{T}} \boldsymbol{C}_{\boldsymbol{x} \boldsymbol{y}} \boldsymbol{\gamma}}{\sqrt{\boldsymbol{\beta}^{\mathrm{T}} \boldsymbol{C}_{\boldsymbol{x} \boldsymbol{x}} \boldsymbol{\beta} \boldsymbol{\gamma}^{\mathrm{T}} \boldsymbol{C}_{\boldsymbol{y} \boldsymbol{y}} \boldsymbol{\gamma}}}
$$

where $\boldsymbol{C}_{\boldsymbol{x} \boldsymbol{y}}, \boldsymbol{C}_{\boldsymbol{x} \boldsymbol{x}}, \boldsymbol{C}_{\boldsymbol{y} \boldsymbol{y}}$ are covariance matrices, $\boldsymbol{\beta}$ and $\boldsymbol{\gamma}$ are the two weight vectors that determine the linear combination of the multidimensional variables $\boldsymbol{x}$ and $\boldsymbol{y}$.

In previous work about CCA based fMRI analysis [7, 8, 9], the two weight vectors were defined as the temporal and the spatial weight vectors for one activity area, e.g. $\boldsymbol{\beta}=$ $\left[\begin{array}{ll}t_{1} & t_{2}\end{array}\right]^{T}, \gamma=\left[\begin{array}{ll}s_{1} & s_{2}\end{array}\right]^{T}$ if there are two temporal basis functions ( $\mathrm{t}$ ) and two spatial basis functions (s). The temporal basis functions are the same as for the GLM (the stimulus paradigm convolved with the hemodynamic response function and its temporal derivative) while the spatial basis functions can be neighbouring pixels [7, 9] or filter responses from a number of filters [8]. 
In this work we modify the spatial weight vector $\gamma$ to contain weights for more than one activity area, e.g. $\gamma=$ $\left[\begin{array}{llll}s_{a 1} & s_{a 2} & s_{b 1} & s_{b 2}\end{array}\right]^{T}$ for two areas $a$ and $b$. Non-local CCA can thereby find the weight vectors that give the highest canonical correlation between the combination of two areas and the stimulus paradigm. Two isotropic Gaussian filters are used for each activity area, an ordinary lowpass filter and its derivative (with respect to the width parameter sigma) [12]. This makes it possible for CCA to combine filters of different size in different parts of the brain, for example a large filter in the primary motor cortex and a small filter in the somatosensory cortex. Our approach to non-local fMRI analysis might seem similar to ordinary correlation analysis for resting state fMRI [5, 13]. In our case the timeseries in the two areas also have to be correlated to the stimulus paradigm, and not only to each other.

One question still remains: How should the two activity areas be selected? The most straight forward approach to deal with this is to simply go through all the pairwise area combinations. One voxel is considered as the reference, the canonical correlation is calculated for all the other voxels and the highest correlation is saved in the reference voxel. This procedure is then repeated for all the brain voxels. There are two problems with this approach. First, the maximum correlation of a large number of area combinations is, by random chance, always likely to be very high and we would get a high correlation in every part of the brain. Second, to investigate all possible combinations would require the calculation of $N_{v}\left(N_{v}-1\right)$ canonical correlations, where $N_{v}$ is the number of brain voxels. For an fMRI dataset with 20000 brain voxels, this gives a total of 400 million canonical correlations. The solution that we propose is to first apply local CCA and then apply nonlocal CCA to the voxels that survive an initial thresholding of the activity map (e.g. half the maximum test value). This significantly reduces the processing time and the probability of always finding a high correlation. The final activity map is then calculated as the maximum of the two activity maps.

To exclude cases when CCA uses more information from the second area than the reference area, the length of the two sub-vectors $\left(\gamma_{\boldsymbol{a}}=\left[\begin{array}{ll}s_{a 1} & s_{a 2}\end{array}\right]^{T}, \gamma_{\boldsymbol{b}}=\left[\begin{array}{ll}s_{b 1} & s_{b 2}\end{array}\right]^{T}\right)$ of the spatial weight vector $\gamma$ are compared. If the weight vector for the reference area, $\gamma_{a}$, is shorter than the weight vector for the second area, $\gamma_{b}$, the correlation is set to zero. It is the reference area, where the found correlation is saved, that should have the highest contribution to the found activity. A distance threshold is also used (e.g. 10 voxels), such that CCA really combines information from different brain areas.

\subsection{Computational complexity}

One problem with CCA based fMRI analysis is how to calculate the significance threshold, as the distribution of canonical correlations is rather complicated. In order to calculate significance thresholds and $\mathrm{p}$-values that are corrected for the

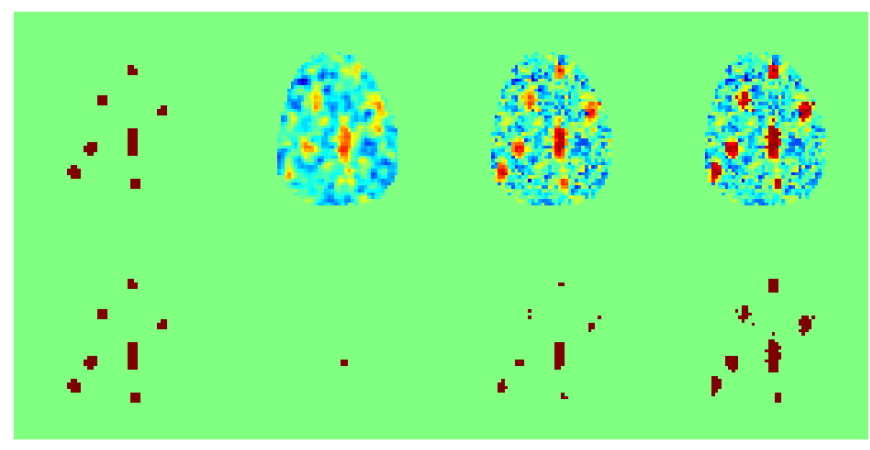

Fig. 1. Top: Original activity maps, normalized between 0 and 1. From left to right: ground truth, GLM, CCA, NLCCA. Bottom: The activity maps thresholded at 0.8.

multiple testing, a random permutation test with 1000 permutations is used, which has been proven to perform better than standard parametric approaches [14]. The double CCA approach (first local CCA, then non-local CCA) is applied in every permutation, each time with an initial threshold calculated from the local CCA approach. If 1000 voxels survive the first activity threshold, one million canonical correlations have to be calculated in each permutation, which gives a total of one billion canonical correlations (if all area combinations would be investigated, 400 billion canonical correlations would have to be calculated). In order to make this practicable, we implemented the algorithm on the graphics processing unit (GPU) $[13,15,16]$. For our multi-GPU implementation with 1440 processor cores, 1000 permutations with NL CCA takes about 3 minutes.

\section{RESULTS}

A comparison between GLM, CCA and NL CCA was made, first on simulated data and then on real data.

\subsection{Simulated data}

In order to compare the different approaches in an objective way, a simulated dataset was created. Activity was put into a number of boxes with varying signal strength. A comparison of the activity maps for the simulated data is given in Figure 1. ROC curves indicated that the non-local approach was better in some cases. The general problem with simulated data, is that it is easy to change the simulation in order to fit a new algorithm.

\subsection{Real data}

Two single subject datasets were also used to compare the algorithms, the test subject was a 50 year old healthy male. The data was collected with a 1.5 T Philips Achieva MR scanner. The following settings were used: repetition time $2 \mathrm{~s}$, echo 
Table 1. A comparison between significance thresholds and the number of significantly active voxels for the three algoritms. M1 stands for left hand activity and M2 stands for right hand activity. $\mathrm{T}$ stands for significance threshold for $\mathrm{p}=0.05$ (corrected for multiple testing) and $\mathrm{S}$ stands for the number of significantly active voxels.

\begin{tabular}{|c|c|c|c|c|}
\hline Algorithm & M1, T & M1, S & M2, T & M2, S \\
\hline GLM & 5.86 & 102 & 5.27 & 74 \\
\hline CCA & 0.65 & 92 & 0.61 & 78 \\
\hline NL CCA & 0.74 & 273 & 0.71 & 147 \\
\hline
\end{tabular}

time $40 \mathrm{~ms}$, flip angle 90 degrees, isotropic voxel size 3.75 $\mathrm{mm}$. A field of view of $240 \mathrm{~mm}$ thereby resulted in slices with $64 \times 64$ pixels, a total of 22 slices were collected every other second. The experiments were $160 \mathrm{~s}$ long, resulting in 80 volumes to be processed. The datasets contain about 20 000 within-brain voxels. Two Motor datasets were used, the subject periodically activated the left or right hand (20 s activity, $20 \mathrm{~s}$ rest). A significance threshold for $\mathrm{p}=0.05$ (corrected for multiple testing) was used. The resulting activity maps are given in Figure 2, an example of combined brain areas is given in Figure 3. The significance thresholds and number of significantly active voxels are given in Table 1 .

\section{DISCUSSION}

We have presented an algorithm for non-local analysis of fMRI data. One obvious idea for future work is to extend the algorithm to more than two activity areas. The principle for three activity areas is the same as for two, but the drawback is that the number of possible area combinations increases exponentially. One solution to this could be to use connectivity information as a prior to which combinations to try [17].

While non-local fMRI analysis can be more sensitive, it will be harder to interpret the activity maps.

\section{ACKNOWLEDGEMENT}

This work was supported by the Linnaeus center CADICS, founded by the Swedish research council, and by the Neuroeconomic research group at Linköping University. The fMRI data was collected at the Center for Medical Image Science and Visualization (CMIV). NovaMedTech is acknowledged for financial support of our GPU hardware.

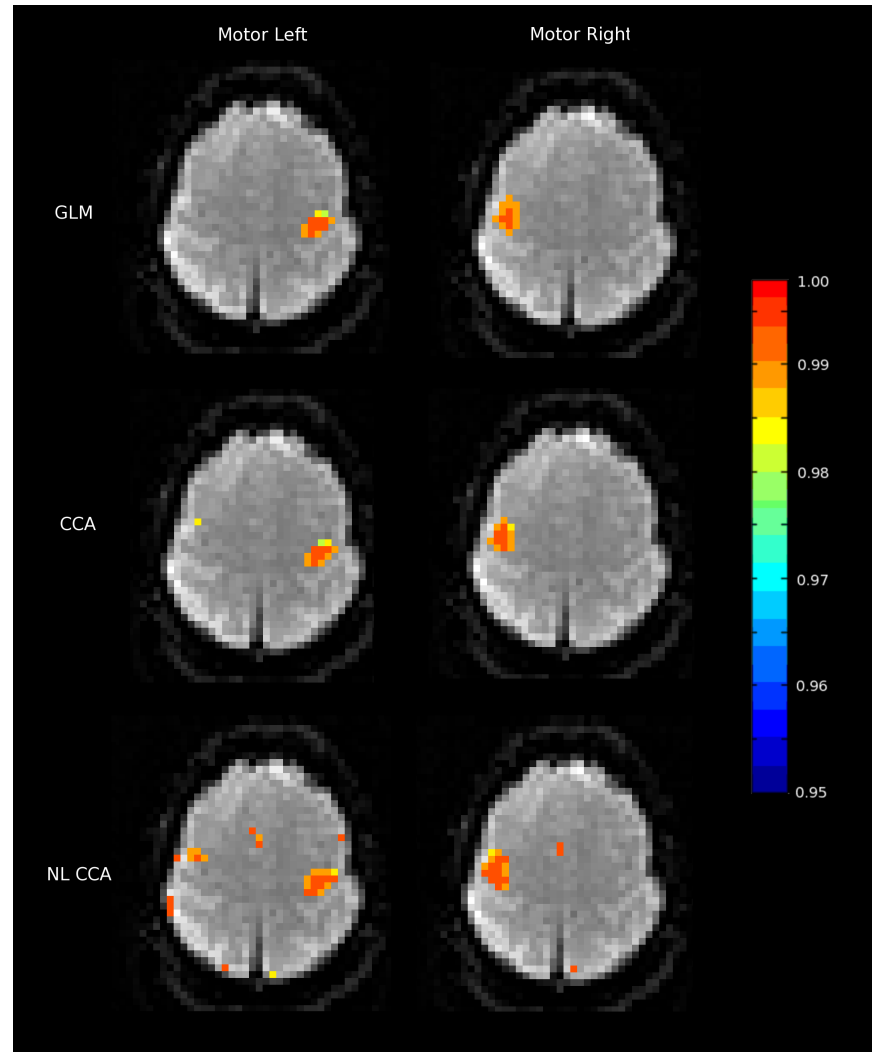

Fig. 2. A comparison of corrected p-values from GLM, CCA and NL CCA, calculated from a random permutation test with 1000 permutations. The activity maps are thresholded at the same significance level, corrected $p=0.05$. The neurological display convention is used (left is left), 1-p is shown instead of $p$. For the left hand activity, NL CCA detects significant activity in the supplementary motor area (SMA), in the visual cortex and in the somatosensory cortex that not is detected by GLM or CCA. For the right hand activity, NL CCA detects significant activity in the SMA and in the visual cortex that not is detected by GLM or CCA. Activity in the visual cortex might seem strange, but this is probably due to the fact that the subject received video instructions through virtual reality goggles (fickering images showing which hand to activate, no flickering during rest). 


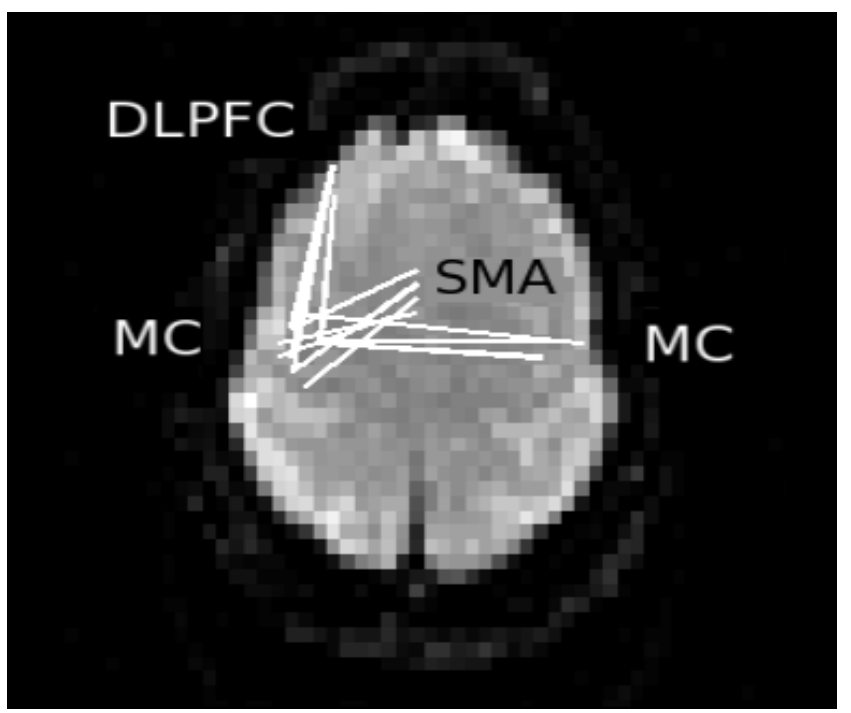

Fig. 3. An example of brain areas that have been combined by the non-local CCA algorithm. By combining information from the left and the right motor cortex $(M C)$, the supplementary motor area (SMA) and the dorsolateral prefrontal cortex (DLPFC), more brain activity can be detected.

\section{REFERENCES}

[1] M.L. Anderson and T. Oates, "A critique of multivoxel pattern analysis," in Proceedings of the 32nd Annual Meeting of the Cognitive Science Society, 2010, pp. 1511-1516.

[2] N. Kriegeskorte, R. Goebel, and P. Bandettini, "Information-based functional brain mapping," PNAS, vol. 103, pp. 3863-3868, 2006.

[3] F. De Martino, G. Valente, N. Staeren, J. Ashburner, R. Goebel, and E. Formisano, "Combining multivariate voxel selection and support vector machines for mapping and classification of fMRI spatial patterns," Neurolmage, vol. 43, pp. 44-58, 2008.

[4] M. Björnsdotter, K. Rylander, and J. Wessberg, "A Monte Carlo method for locally multivariate brain mapping," NeuroImage, vol. 56, pp. 508-516, 2011.

[5] B. Biswal, F.Z. Yetkin, V.M. Haughton, and J.S. Hyde, "Functional connectivity in the motor cortex of resting state human brain using echo-planar MRI," Magnetic Resonance in Medicine, vol. 34, pp. 537-541, 1995.

[6] H. Hotelling, "Relation between two sets of variates," Biometrika, vol. 28, pp. 322-377, 1936.

[7] O. Friman, J. Carlsson, P. Lundberg, M. Borga, and H. Knutsson, "Detection of neural activity in functional MRI using canonical correlation analysis," Magnetic
Resonance in Medicine, vol. 45, no. 2, pp. 323-330, 2001.

[8] O. Friman, M. Borga, P. Lundberg, and H. Knutsson, "Adaptive analysis of fMRI data," NeuroImage, vol. 19, pp. 837-845, 2003.

[9] R. Nandy and D. Cordes, "A novel nonparametric approach to canonical correlation analysis with applications to low CNR functional MRI data," Magnetic Resonance in Medicine, vol. 49, pp. 1152-1162, 2003.

[10] A. Buades, B. Coll, and J.M. Morel, "A non-local algorithm for image denoising," in IEEE International Conference on Computer Vision and Pattern Recognition, 2005, pp. 60-65.

[11] K.J. Friston, A.P. Holmes, K.J. Worsley, J.B. Poline, C. Frith, and R.S.J. Frackowiak, "Statistical parametric maps in functional imaging: A general linear approach," Human Brain Mapping, vol. 2, pp. 189-210, 1995.

[12] O. Friman, "Subspace models for functional MRI data analysis," in Proceedings of IEEE International Symposium on Biomedical Imaging (ISBI), 2004, pp. 1-4.

[13] A. Eklund, O. Friman, M. Andersson, and H. Knutsson, "A GPU accelerated interactive interface for exploratory functional connectivity analysis of fMRI data," in IEEE International Conference on Image Processing (ICIP), 2011, pp. 1621-1624.

[14] A. Eklund, M. Andersson, C. Josephson, M. Johannesson, and H. Knutsson, "Does parametric fMRI analysis with SPM yield valid results? - An empirical study of 1484 rest datasets," NeuroImage, http://dx.doi.org/10.1016/j.neuroimage.2012.03.093, 2012.

[15] A. Eklund, M. Andersson, and H. Knutsson, "Fast random permutation tests enable objective evaluation of methods for single subject fMRI analysis," International Journal of Biomedical Imaging, Article ID 627947, 2011.

[16] Anders Eklund, Mats Andersson, and Hans Knutsson, "fMRI analysis on the GPU - possibilities and challenges," Computer Methods and Programs in Biomedicine, vol. 105, pp. 145-161, 2012.

[17] B. Ng, R. Abugharbieh, G. Varoquaux, J.B. Poline, and B. Thirion, "Connectivity-informed fMRI activation detection," Lecture notes in computer science, Proceedings of the 14th International conference on Medical image computing and computer-assisted intervention (MICCAI), vol. 6892, pp. 285-292, 2011. 